\title{
Efficacy and Safety of Focused Low-Intensity Pulsed Ultrasound Therapy Versus Pulsed Shortwave Diathermy for the Management of Knee Osteoarthritis: A Randomized Controlled Trial
}

\author{
Lang Jia ( $\sim$ jialang@hospital.cqmu.edu.cn ) \\ the Second Affiliated Hospital of Chongqing Medical University \\ Dongqian Li \\ the Second Affiliated Hospital of Chongqing Medical University \\ Xia Wei \\ the Second Affiliated Hospital of Chongqing Medical University \\ Jinyun Chen \\ Chongqing Medical University \\ Deyu Zuo \\ Chongqing Traditional Chinese Medicine Hospital \\ Wenzhi Chen \\ the Second Affiliated Hospital of Chongqing Medical University
}

\section{Research Article}

Keywords: Randomized controlled trial, knee osteoarthritis, focused low-intensity pulsed ultrasound, pulsed shortwave diathermy, pain

Posted Date: January 17th, 2022

DOI: https://doi.org/10.21203/rs.3.rs-1236940/v1

License: (c) (i) This work is licensed under a Creative Commons Attribution 4.0 International License. Read Full License 


\section{Abstract}

Focused low-intensity pulsed ultrasound(FLIPUS)and pulsed shortwave diathermy (PSWD) are presented as possible solution to attenuate the loss of function in the elderly with knee osteoarthritis(OA). The question remains unanswered which one is better modalities. The aim of this study was to compare the efficacy and safety of FLIPUS with PSWD in subjects with painful knee OA. In a prospective randomized trial,114 knee OA patients were randomly allocated to receive FLIPUS or PSWD therapy. The primary outcome was the change from baseline in the Western Ontario and McMaster Universities Osteoarthritis Index (WOMAC) total and subscale scores. Secondary outcomes included numerical rating scale (NRS), Time Up and Go (TUG) test, active joint range of motion (ROM), and Global Rating of Change (GRC) scale. Data were collected at baseline, 12 days, 12 weeks and 24 weeks. Patients receiving FLIPUS therapy experienced significantly greater improvements in WOMAC total scores than patients receiving PSWD therapy at 12 days post-therapy (mean difference, $-10.50 ; 95 \% \mathrm{Cl},-13.54$ to $-7.45 ; P=$ 0.000). The results of the NRS scores, TUG test, ROM and GRC scale showed that participants treated with FLIPUS reported less pain and better physical function and health status than those treated with PSWD $(P=0.011, P$ $=0.005, P=0.025, P=0.011$, respectively). Furthermore, patients in the FLIPUS group showed significant improvements in the WOMAC total scores and NRS scores at 12 and 24 weeks of follow-up $(P=0.000$ for all). FLIPUS immediately improved knee pain, dysfunction and health status compared with PSWD. In addition, the benefit of FLIPUS persists throughout follow-up.

Trial registration: Chinese Clinical Trial Registry, ChiCTR2000032735. Registered 08/05/2020, http://www.chictr.org.cn/showproj.aspx? proj= 53413.

\section{Introduction}

The pain and knee dysfunctionality associated with knee osteoarthritis $(\mathrm{OA})$ are not always readily controlled and improved. In recent years, the mechanical effects of focused low-intensity pulsed ultrasound(FLIPUS) and the thermal effects of pulsed shortwave diathermy(PSWD) have been therapeutic options in mild to moderate knee $\mathrm{OA}^{1}$. Both modalities aimed at the management of knee $\mathrm{OA}$ are in part due to relieved pain, an increased range of motion, accelerated tissue repair, reduced edema and inability and, if possible, slowing its progression ${ }^{2,3}$, and therefore are conditionally recommended for patients with knee OA by clinical guideline ${ }^{4}$. Assuming a comparable indication and efficacy of both FLIPUS and PSWD, the question remains unanswered whether one of the two modalities should be preferred.

Previous studies directly compared traditional non-focused therapeutic ultrasound with shortwave diathermy ${ }^{5,6}$. However, there was no significant difference between the two modalities in terms of pain reduction and functional improvement ${ }^{6}$. We speculated that the same biological action (thermal effects) and therapeutic targets (muscles and tendons) of the traditional ultrasound and shortwave diathermy led to similar effectiveness in the management of knee OA.

To date, only one result has been obtained on FLIPUS in the context of knee OA ${ }^{3}$. No clinical trial has directly compared the effects of mechanical effects of FLIPUS and thermal effects of PSWD in patients with mild to moderate knee OA. The aim of this study was therefore to compare both the safety and clinical effects of a twelveday intervention with each device in a population with knee OA. 
Primarily, the improvement of knee functional ability by each modality was examined and compared. A range of patient-reported outcome measures for knee pain, knee functional tasks and health-related quality of life were evaluated to allow application in the clinical setting.

\section{Materials And Methods}

Study Design and participants. This pilot study was designed according to the CONSORT 2010 statement. This was a prospective, randomized-controlled, observer blinded study with 24-week follow-up done between June 2020 and December 2020. Subjects were recruited through advertisements placed in a rehabilitation department of the Second Affiliated Hospital of Chongqing Medical University. The inclusion and exclusion criteria are shown in Table 1. All subjects gave written informed consent before participating in the study. The study was approved by the Ethics Committee of the Second Affiliated Hospital, Chongqing Medical University. The Chinese Clinical Trial Registry granted full approval of the study protocol, recruitment materials, and consent form (URL:

https://www.chictr.org.cn; unique identifier: ChiCTR2000032735; date of registration: 08/05/2020). The procedures conformed with good clinical practice and with the ethical standards laid down in the 1964 Declaration of Helsinki and its later amendments.

Table 1

Inclusion and exclusion criteria.

\section{Inclusion criteria}

Age 40-80 years (either sex)

Met the criteria for the ACR clinical classification of knee OA

Had radiographic evidence of knee OA (weight-bearing views) assessed as Kellgren-Lawrence grade I to grade III

Had average knee pain $\geq 3$ on an 11-point NRS in the past week

\section{Exclusion criteria}

Knee pain caused by other diseases (rheumatoid arthritis, gouty arthritis, infectious arthritis)

A history of knee joint replacement on the study knee; current or past (within 6 months) oral or intra-articular corticosteroid use

Physiotherapy, acupuncture treatment, the use of exercises specifically for the knee within the past 6 months

A medical condition that precludes safe exercise (such as uncontrolled hypertension, a heart condition, hematological diseases coagulopathy, gastrointestinal ulcers, or a hemorrhage)

A history of taking NSAIDs or symptomatic slow-acting drugs for OA (diacerein, hyaluronic acid) within the previous 30 days

The inability to complete the study.

ACR American College of Rheumatology, OA osteoarthritis, NRS Numerical Rating Scale, NSAIDs nonsteroidal anti-inflammatory drugs. 
Table 2 The baseline demographics and clinical features of patients.

\begin{tabular}{|c|c|c|c|}
\hline Variable & $\begin{array}{l}\text { FLIPUS } \\
(n=57)\end{array}$ & $\begin{array}{l}\text { PSWD } \\
(n=57)\end{array}$ & $P$ value \\
\hline Age, years & $62.28 \pm 10.88$ & $59.93 \pm 8.97$ & 0.211 \\
\hline Women, no. (\%) & $42(73.68 \%)$ & $45(78.95 \%)$ & 0.660 \\
\hline $\mathrm{BMI}, \mathrm{kg} / \mathrm{m}^{2}$ & $25.18 \pm 3.26$ & $25.29 \pm 2.85$ & 0.854 \\
\hline Duration of disease, months & $120.32 \pm 74.88$ & $118.54 \pm 89.73$ & 0.909 \\
\hline \multicolumn{4}{|c|}{ Kellgren-Lawrence grade, no. (\%) } \\
\hline Grade I & $4(7.01 \%)$ & $6(10.53 \%)$ & \multirow[t]{3}{*}{0.205} \\
\hline Grade II & $40(70.18 \%)$ & $45(78.94 \%)$ & \\
\hline Grade III & $13(22.81 \%)$ & $6(10.53 \%)$ & \\
\hline \multicolumn{4}{|l|}{ Primary Outcome } \\
\hline \multicolumn{4}{|l|}{ WOMAC } \\
\hline Total & $34.49 \pm 10.26$ & $33.70 \pm 7.91$ & 0.646 \\
\hline Pain & $6.40 \pm 2.15$ & $7.44 \pm 2.61$ & 0.551 \\
\hline Stiffness & $2.35 \pm 1.13$ & $2.56 \pm 1.02$ & 0.558 \\
\hline Physical Function & $23.89 \pm 8.37$ & $25.54 \pm 6.58$ & 0.688 \\
\hline \multicolumn{4}{|l|}{ Secondary Outcomes } \\
\hline NRS & $5.51 \pm 1.28$ & $5.81 \pm 1.06$ & 0.825 \\
\hline GROC score & $-2(2)$ & $-1(3)$ & 0.066 \\
\hline ROM, degree & $127.39 \pm 5.92$ & $126.23 \pm 6.57$ & 0.895 \\
\hline TUG test & $12.91 \pm 2.45$ & $12.60 \pm 2.33$ & 0.296 \\
\hline \multicolumn{4}{|c|}{$\begin{array}{l}\text { FLIPUS focused low-intensity pulsed ultrasound, } P S W D \text { pulsed shortwave diathermy, BMI body mass index, } \\
\text { WOMAC Western Ontario and McMaster Universities Osteoarthritis Index, NRS Numerical Rating Scales, GROC } \\
\text { Global Rating of Change scale, IQR interquartile range, ROM range of motion; TUG Timed Up and Go test. } \\
\text { Measurement data are represented as mean } \pm \text { SD (Normally distributed data) or median (IQR) (Non-normally } \\
\text { distributed data). Enumeration data are represented as frequencies (proportion). }\end{array}$} \\
\hline
\end{tabular}


Table 3 Primary and secondary efficacy measures after 12 days of treatment.

\begin{tabular}{|c|c|c|c|c|}
\hline Variable & FLIPUS & PSWD & $\begin{array}{l}\text { Mean Between-Group } \\
\text { Difference: } \\
\text { FLIPUS-PSWD }(95 \% \mathrm{Cl})\end{array}$ & $l_{\text {value }}$ \\
\hline $\mathrm{n}$ & 57 & 57 & & \\
\hline \multicolumn{5}{|l|}{ Primary Outcome } \\
\hline \multicolumn{5}{|l|}{ WOMAC } \\
\hline Total & $17.86 \pm 9.77$ & $28.35 \pm 7.25$ & $-10.50(-13.54,-7.45)$ & 0.000 \\
\hline $\begin{array}{l}\text { Change }(95 \% \mathrm{Cl}) \text { from } \\
\text { baseline }\end{array}$ & $\begin{array}{l}-16.63(-17.85 \\
-15.42)\end{array}$ & $\begin{array}{l}-5.35(-6.00 \\
-4.71)\end{array}$ & & \\
\hline Pain & $3.07 \pm 1.61$ & $4.70 \pm 2.13$ & $-1.63(-2.30,-0.96)$ & 0.005 \\
\hline $\begin{array}{l}\text { Change }(95 \% \mathrm{Cl}) \text { from } \\
\text { baseline }\end{array}$ & $-3.33(-3.69,-2.98)$ & $\begin{array}{l}-2.74(-3.03 \\
-2.44)\end{array}$ & & \\
\hline Stiffness & $0.70 \pm 0.76$ & $1.88 \pm 0.93$ & $-1.18(-1.46,-0.89)$ & 0.000 \\
\hline $\begin{array}{l}\text { Change }(95 \% \mathrm{Cl}) \text { from } \\
\text { baseline }\end{array}$ & $-1.65(-1.93,-1.36)$ & $\begin{array}{l}-0.68(-0.88 \\
-0.49)\end{array}$ & & \\
\hline Physical Function & $13.82 \pm 8.29$ & $22.04 \pm 6.42$ & $-8.21(-11.13,-5.29)$ & 0.000 \\
\hline $\begin{array}{l}\text { Change }(95 \% \mathrm{Cl}) \text { from } \\
\text { baseline }\end{array}$ & $-10.07(-11.38,-8.76)$ & $\begin{array}{l}-3.51(-4.48 \\
-2.54)\end{array}$ & & \\
\hline \multicolumn{5}{|l|}{ Secondary Outcome } \\
\hline NRS & $1.89 \pm 1.01$ & $2.65 \pm 1.01$ & $-0.76(-1.12,-0.39)$ & 0.011 \\
\hline $\begin{array}{l}\text { Change }(95 \% \mathrm{Cl}) \text { from } \\
\text { baseline }\end{array}$ & $-3.61(-3.85,-3.37)$ & $\begin{array}{l}-3.16(-3.36 \\
-2.95)\end{array}$ & & \\
\hline GRC score & $+4(2)$ & $+3(2)$ & & 0.011 \\
\hline ROM, degree & $130.56 \pm 4.65$ & $128.30 \pm 6.24$ & $2.26(0.21,4.32)$ & 0.025 \\
\hline $\begin{array}{l}\text { Change }(95 \% \mathrm{Cl}) \text { from } \\
\text { baseline }\end{array}$ & $3.16(2.41,3.94)$ & $2.07(1.50,2.64)$ & & \\
\hline TUG test & $10.61 \pm 2.29$ & $11.84 \pm 2.42$ & $-1.23(-2.02,-0.44)$ & 0.005 \\
\hline $\begin{array}{l}\text { Change }(95 \% \mathrm{Cl}) \text { from } \\
\text { baseline }\end{array}$ & $-2.30(-2.63,-1.97)$ & $\begin{array}{l}-0.75(-0.91 \\
-0.60)\end{array}$ & & \\
\hline \multicolumn{5}{|c|}{$\begin{array}{l}\text { FLIPUS focused low-intensity pulsed ultrasound, } P S W D \text { pulsed shortwave diathermy, } B M / \text { body mass index, } \\
\text { WOMAC Western Ontario and McMaster Universities Osteoarthritis Index, NRS Numerical Rating Scales, GROC } \\
\text { Global Rating of Change scale, IQR interquartile range, ROM range of motion; TUG Timed Up and Go test, CI } \\
\text { confidence interval. Measurement data are represented as mean } \pm \text { SD (Normally distributed data) or median } \\
\text { (IQR) (Non-normally distributed data). }\end{array}$} \\
\hline
\end{tabular}


Table 4 WOMAC total scores and NRS score measures after 12 and 24 weeks of follow-up.

\begin{tabular}{|c|c|c|c|c|c|c|c|c|}
\hline & \multicolumn{4}{|c|}{ WOMAC (Total scores) } & \multicolumn{4}{|l|}{ NRS scores } \\
\hline & Baseline & 12 weeks & 24 weeks & $\begin{array}{l}P \\
\text { value }\end{array}$ & Baseline & 12 weeks & 24 weeks & $\begin{array}{l}P \\
\text { value }\end{array}$ \\
\hline FLIPUS & $34.49 \pm 10.26$ & $22.89 \pm 9.32$ & $28.02 \pm 9.29$ & 0.000 & $5.70 \pm 1.24$ & $3.05 \pm 0.99$ & $4.91 \pm 0.89$ & 0.000 \\
\hline PSWD & $33.70 \pm 7.91$ & $30.46 \pm 6.77$ & $34.98 \pm 6.72$ & 0.000 & $5.61 \pm 1.13$ & $4.84 \pm 0.94$ & $6.28 \pm 0.96$ & 0.000 \\
\hline$P$ value & 0.646 & 0.000 & 0.000 & & 0.825 & 0.000 & 0.000 & \\
\hline
\end{tabular}

The study consisted of a screen visit, a baseline visit-during which FIPUS or PSWD was done- and follow-up visits at 12 days, 12 and 24 weeks post-therapy. Potential study participants returned for a baseline visit after a 1-week washout period for nonsteroidal anti-inflammatory drugs (NSAIDs) and analgesics. Before randomization, demographic data and baseline assessments were collected.

Randomization procedures. Enrolled patients were randomized (1:1) to 2 groups. Opaque randomization envelopes with sequentially numbered allocation were generated by a person who was not clinically involved in the study. When a patient consented to the trial, the patient selected one of the envelopes and then was given the allocated therapy ${ }^{7}$.

Intervention. The patients in the FLIPUS group received focused low-intensity pulsed ultrasound therapy (The Model CZG200 Ultrasound Therapeutic Device for Arthritis used, Chongqing Haifu Medical Technology Co. Ltd., China) for 20 minutes once daily for a total treatment duration of 12 days ${ }^{3}$. The device had an ultrasonic transducer diameter of $25 \mathrm{~mm}$, a radius-of-curvature of $28 \mathrm{~mm}$, a frequency of 0.6 megaherz $(\mathrm{MHz})$, a pulse repetition frequency of 300 herz $(\mathrm{Hz})$, and a spatial and temporal average intensity (Ista) of 120 milliwatts $(\mathrm{mW}) / \mathrm{cm}^{2}$, and a duty cycle of $20 \%$ 3 . All treatments were standardized using a device that placed the participant in a sitting position, and the knee was angled $\sim 90^{\circ}$ in the flexion position. The four ultrasound probes were close to the surface skin of the EX-LE 4 acupoint (located in the depression medial to the patellar ligament when the knee was flexed), ST 35 acupoint (located in the depression lateral to the patellar ligament when the knee was flexed), and medial and lateral knee joint spaces, respectively, as previously described ${ }^{3}$ (Fig. 1).

The patients in the PSWD group received pulsed shortwave diathermy therapy(The Curapulse 970 model, EnrafNonius, Rotterdam, the Netherlands) for 20 minutes once daily for a total treatment duration of 12 days ${ }^{7}$. The machine's program was set at 'chronic OA model' and delivered electromagnetic waves with a frequency of 27.12 $\mathrm{MHz}$, a pulse repetition frequency of $300 \mathrm{~Hz}$, a pulse duration of $300 \mu \mathrm{s}$, a peak power of 200 watts (W), and a mean power of $18 \mathrm{~W}^{8}$. All patients reported a mild but comfortable feeling of warmth.

Patients with symptoms in both knees received treatment in both knees, but trial outcomes were assessed only in the knee with worse symptoms ${ }^{8}$. 
Outcomes Measures. The primary outcome measures were functional capacity, assessed by the Western Ontario and McMaster Universities Osteoarthritis Index (WOMAC) ${ }^{9}$. The Minimal Clinically Important Difference for knee OA is -7.9 points for WOMAC total score ${ }^{10}$. Secondary outcomes included the following: (1) Numerical rating scale (Minimal Clinically Important Difference, $\geq 15 \%$ improvements from baseline), (2) Time Up and Go test (Minimal Clinically Important Difference, 1.14 seconds $^{11}$ ), (3) Active joint range of motion, and (4) Global Rating of Change scale (Minimal Clinically Important Difference, a score of +3 or higher ${ }^{12}$ ). All of them were evaluated at baseline, after 12 days of treatment, and at follow-up after 12 and 24 weeks.

Statistical analysis. The statistical evaluation was carried out after qualified statistical advice. Case number planning was calculated for the primary outcome measure and the reduction in WOMAC total score compared to baseline. Based on a study by Tubach et al $^{10}$., who found a reduction of 7.9 points with the WOMAC total score (effect size of 0.59 ), the case number was calculated using a t test-based model in $\mathrm{G}$ * Power version $3.1 .5^{13}$. A power of 0.8 with a significance level of 0.05 was chosen and resulted in a required number of 47 cases. We added approximately $10 \%-20 \%$ more participants to account for potential loss to follow-up, resulting in a final enrollment goal of 114 participants (57 per group).

Independent t tests (continuous variables), Mann-Whitney U tests (ordinal variables), and chi-square tests (nominal variables) were used to compare the characteristics of the FLIPUS group and PSWD group.

All outcomes were analyzed using the intention-to-treat approach. We tested the data normality and sphericity using the Shapiro-Walk test and Mauchly's test, respectively. Repeated measures analysis of covariance (ANCOVA) was used to compare the WOMAC score, Numerical Rating Scale at baseline, after 12 days of treatment, and at follow-up after 12 and 24 weeks, as well as the Time Up and Go test, joint range of motion, and Global Rating of Change scale at baseline and after 12 days of treatment.

Post hoc analyses were conducted within (using paired t tests for comparisons across time) and between (using independent $t$ tests within each time point) groups via pairwise comparisons with Bonferroni correction. ANCOVA using a last-observation-carried forward (LOCF) approach for the imputation of missing data was applied as a supportive analysis.

All statistical analyses were performed using SPSS version 20 (SPSS Inc., Chicago, IL, USA) with a global alpha of 0.05 .

\section{Results}

Patient characteristics. A total of 130 patients were screened in this study. After 16 patients were excluded, 114 were evenly distributed between the two arms. A total of ten (8.8\%) patients dropped out during the trial: four (7.0\%) in the FLIPUS group and six (10.5\%) in the PSWD group (Fig.2).

The baseline characteristics of the sample are shown in Table 2. No differences were found between the groups at the beginning of the trial. There were no losses during the intervention period.

Primary and secondary outcomes. Pre-and postintervention primary and secondary outcome comparisons between FLIPUS and PSWD are shown in Table 3. Initial values, final values and the difference between them were compared in the statistical analysis. 
There was a statistically significant improvement in both groups according to the WOMAC total scores after 12 days of treatment compared with the baseline scores. However, the PSWD group did not exhibit an improvement from baseline of -7.9 points in the WOMAC total score after 12 days of treatment. Specifically, 4 patients (7.02\%) in the FLIPUS group and 22 patients (38.60\%) in the PSWD group failed to reach the Minimal Clinically Important Difference for the WOMAC total score. Additionally, FLIPUS-treated patients experienced statistically significantly greater pain reduction, physical function and stiffness improvement than PSWD-treated patients according to the WOMAC subscale scores after 12 days of treatment ( $P=0.005, P=0.000$ and $P=0.000$, respectively).

A significantly greater percentage of FLIPUS-treated patients, compared to PSWD-treated patients, experienced a greater reduction in pain from baseline according to the Numerical Rating Scale score. Both groups had an improvement from baseline of at least 15\% according to the Numerical Rating Scale scores after 12 days of treatment. However, patients in the FLIPUS group showed better analgesia effects than PSWD $(P=0.011)$.

Patients in the FLIPUS group performed better knee functional tasks (had lower mean times) according to the Time Up and Go test than patients in the PSWD group ( $P=0.005)$. Notably, the FLIPUS group had an improvement from baseline of at least 1.14 seconds in the Time Up and Go test after 12 days of treatment. However, the PSWD group failed to reach a clinically important improvement according to the Time Up and Go test.

There was a significant difference between the two groups with respect to the range of knee motion after 12 days of intervention $(P=0.025)$, and the total mean increment of range of knee motion was greater in the FLIPUS group than in the PSWD group $(P=0.000)$.

Both groups were considered to be clinically meaningful according to the Global Rating of Change scale after 12 days of treatment. However, a total of 15 patients (26.32\%) in the FLIPUS group, compared with 26 (45.61\%) in the PSWD group, did not have a score of +3 or higher on the Global Rating of Change scale after 12 days of treatment.

Table 4 summarizes the results of clinical outcomes at follow-ups. The functional ability (WOMAC total score) and movement pain (Numerical Rating Scale score) measures remained significantly lower at the 12-week and 24-week follow-ups than at baseline in the FLIPUS group but were higher at the 24-week follow-up than at baseline in the PSWD group $(P=0.000)$.

No adverse effects (mild pain, swelling and feeling of vasodilatation, etc.) were observed in both groups in the present trial.

\section{Discussion}

This study compared FLIPUS with PSWD in symptomatic patients with clinical and radiographic evidence of knee $\mathrm{OA}$ and showed that the immediate posttreatment pain relief expected with PSWD can also be obtained with FLIPUS therapy; however, pain relief and physical function improvements from FLIPUS therapy persist for 24 weeks. We performed sensitivity analyses and subgroup analysis, and the results did not favor PSWD in the improvement of physical function. To the best of our knowledge, this is the first study to compare the therapeutic effects of FLIPUS with PSWD on patients with knee OA.

To date, only a few and partly controversial results have been obtained on both ultrasound and PSWD in the context of knee OA. In previous studies, unfocused continuous ultrasonic waves with frequencies of 1 or $1.5 \mathrm{MHz}^{14-}$ ${ }^{19}$ and $1 \varangle 2.5 \mathrm{~W} / \mathrm{cm}^{214-19}$ were applied for tendons and muscles around the knee joint ${ }^{15,17,19}$. The biological effects 
of ultrasound are mainly considered to be thermal, which promotes blood circulation and alleviates spasms in various muscles and tendons ${ }^{18}$. In the last decade, studies have demonstrated that PSWD may also induce an elevation of tissue temperature that is dependent on the total average power delivered ${ }^{20,21}$ and significant physiological thermal effects on blood volume and skin temperature, which may induce vasodilatation, elevation of pain threshold, reduction in muscle spasm, acceleration of cellular activity, and increased soft tissue extensibility 22,23 .

Unsurprisingly, the same biological action (thermal effects) and therapeutic targets (muscles and tendons)of unfocused continuous ultrasound and PSWD led to similar effectiveness in the management of knee OA, which may explain why no significant differences were concluded between unfocused continuous ultrasound and shortwave diathermy ${ }^{5,6}$. However, the thermal effects of ultrasound and PSWD for alleviating muscle and tendon spasms around the joints are insufficiency. Muscle does not absorb energy well because of its homogeneity, high water content, and low collagen content, and heating muscles and tendons involves treating a larger area than unfocused ultrasound, and PSWD can heat effectively ${ }^{18}$, which may explain why only a temporal pain reduction effect was concluded after ultrasound or PSWD application.

Recently, our previous in vivo experiment ${ }^{24}$ demonstrated that the main biological effects of FLIPUS are mechanical, which could be quite different from the unfocused continuous ultrasound reported in other previous studies (thermal effects). In addition to the different biological effects of FLIPUS, selecting the targets for energy application is another key consideration with treatment. We also revealed that FLIPUS at $0.6 \mathrm{MHz}$ could propagate through the patella and soft tissue to stimulate the cartilage directly, which could be quite different from the therapeutic targets of unfocused continuous ultrasound and PSWD reported in other previous studies (tendons and muscles around the knee joint). The potential mechanism of FLIPUS included promoting extracellular matrix reservation, decreasing the joint effusion volume, proinflammatory mediators, and cell apoptosis, and inducing cell proliferation $^{24}$.

In the current study, the Numerical Rating Scale pain scores improved in both groups, which is consistent with an earlier trial, systematic review and meta-analysis ${ }^{3,25}$. However, the reduction in the Numerical Rating Scale scores was greater in the FLIPUS group than in the PSWD group after 12 days of intervention and during the follow-up period. In addition, the PSWD group failed to reach the Minimal Clinically Important Difference at the 12-week and 24-week follow-ups, which indicates that the pain reduction effect in the PSWD group was noticed primarily immediately posttreatment, and the analysis yielded no short-term follow-up effect.

The improvement in the WOMAC total score from baseline failed to reach the Minimal Clinically Important Difference in the PSWD group. Furthermore, although the improvements in physical function and disability status according to the Time Up and Go test and range of knee motion were different in both groups after treatment compared with baseline, the improvement in the Time Up and Go test in the PSWD group failed to reach the Minimal Clinically Important Difference. Based on the present results, PSWD does not improve physical function or disability status in patients with knee OA, which is consistent with an earlier trial[11]. We hypothesize that the improved function and disability were attributable to the better analgesia effects of ultrasonic waves than PSWD.

With respect to the screen on self-perceived changes in health status, the median scores on the Global Rating of Change scale in both groups were above the clinically meaningful threshold of a perceived improvement, which indicated that both modalities could improve health status in symptomatic patients with knee OA. However, FLIPUS improved health status compared with PSWD. 
Finally, no adverse effects occurred during or after FLIPUS and PSWD treatment in the current study; therefore, both modalities can be used safely in patients with knee OA.

The present study has some limitations that should be cautiously considered. First, all participants included in this study were enrolled from a single center, and the relatively small number of cases must be considered. Therefore, future studies with larger populations, a multicenter clinical trial and crossover study design are needed. Second, we did not test OA biomarkers in synovial fluid. Bone morphogenetic protein-2, CTX-II and cartilage oligomeric matrix protein may be promising for predicting the prognosis and progression of OA and should be studied after intervention. Finally, FLIPUS resulted in much better clinical outcomes than PSWD in the current study but likely at a higher cost, contemporary cost-effectiveness analyses that compare these 2 treatments are warranted.

In conclusion, the results from the present study demonstrate that FLIPUS for knee OA resulted in better absolute scores on scales of pain, physical function and health status than PSWD. FLIPUS can therefore be considered a superior resource in the treatment armamentarium of clinicians for patients with knee OA.

\section{Declarations}

\section{Acknowledgements}

We would like to thank Dr Gerry Warner for assistance in editing the manuscript.

\section{Author Contributions}

W.Z.C. conceived and designed the experiments. D.Q.L., D.Y.Z. and X.W. performed the experiments. J.Y.C. performed the statistical analysis. L.J. contributed to drafting of the manuscript. L.J. helped in obtaining funding. W.Z.C. contributed to study supervision. All authors reviewed the manuscript.

\section{Funding}

The author(s) disclosed receipt of the following financial support for the research, authorship and/or publication of this article: This work was supported in part by the National Natural Science Foundation of China (Grant No. 81802234), the Natural Science Foundation of Chongqing (Grant No.cstc2020jcyj-msxmX0134), the Traditional Chinese Medicine Science and Technology Project of Chongqing(Grant No.ZY201802104). Lang Jia was supported by Kuanren Talents Program of the second affiliated hospital of Chongqing Medical University.

\section{Competing interests}

The authors declare no competing interests.

\section{Data Availability Statement}

All data generated or analysed during this study are included in this article. Further enquiries can be directed to the corresponding author.

\section{Ethical approval.}

The study was approved by the institutional review board for human investigation and followed the Declaration of Helsinki, 1996. The study was registered at Chinese Clinical Trial Registry (ChiCTR2000032735, date of registration: 


\section{References}

1. Wang, H. et al. Effects of short-wave therapy in patients with knee osteoarthritis: a systematic review and metaanalysis. Clin Rehabil.31,660-71(2017).

2. Wu, Y. et al. Effects of therapeutic ultrasound for knee osteoarthritis: a systematic review and meta-analysis. Clin Rehabil.33,1863-1875(2019).

3. Jia, L., Wang, Y., Chen, J. \&Chen, W. Efficacy of focused low-intensity pulsed ultrasound therapy for the management of knee osteoarthritis: a randomized, double blind, placebo-controlled trial. Sci Rep. 17,6:35453(2016).

4. Cetin, N., Aytar, A., Atalay, A. \& Akman, M.N. Comparing hot pack, short-wave diathermy, ultrasound, and TENS on isokinetic strength, pain, and functional status of women with osteoarthritic knees: a single-blind, randomized, controlled trial. Am J Phys Med Rehabil. 8,443-451(2008).

5. Boyaci, A., Tutoglu, A., Boyaci, N., Aridici, R. \& Koca, I. Comparison of the efficacy of ketoprofen phonophoresis, ultrasound, and short-wave diathermy in knee osteoarthritis. Rheumatol Int.33,2811-2818 (2013).

6. Jan, M.H. \& Lai, J.S. The effects of physiotherapy on osteoarthritic knees of females. J Formos Med Assoc. 90,1008-1013 (1991).

7. Sun, S.F. et al. Comparing efficacy of intraarticular single crosslinked Hyaluronan (HYAJOINT Plus) and platelet-rich plasma (PRP) versus PRP alone for treating knee osteoarthritis. Sci Rep. 11,140(2021).

8. Laufer, Y., Zilberman, R., Porat, R. \& Nahir, A.M. Effect of pulsed short-wave diathermy on pain and function of subjects with osteoarthritis of the knee: a placebo-controlled double-blind clinical trial. Clin Rehabil.19,255263(2005).

9. Bellamy, N., Buchanan, W.W., Goldsmith, C.H., Campbell, J. \&Stitt, L.W. Validation study of WOMAC: a health status instrument for measuring clinically important patient relevant outcomes to antirheumatic drug therapy in patients with osteoarthritis of the hip or knee. J Rheumatol.15,1833-1840 (1988).

10. Tubach, F. et al. Evaluation of clinically relevant changes in patient reported outcomes in knee and hip osteoarthritis: the minimal clinically important improvement. Ann Rheum Dis. 64,29-33 (2005).

11. Alghadir, A., Anwer, S. \&Brismée, J.M. The reliability and minimal detectable change of Timed Up and Go test in individuals with grade 1-3 knee osteoarthritis. BMC Musculoskelet Disord.16,174(2015).

12. Jaeschke, R., Singer, J. \&Guyatt, G.H. Measurement of health status. Ascertaining the minimal clinically important difference. Control Clin Trials. 10,407-415(1989).

13. Faul, F., Erdfelder, E., Lang, A.G. \& Buchner, A. G*Power 3: a flexible statistical power analysis program for the social, behavioral, and biomedical sciences. Behav Res Methods. 39,175-191 (2007).

14. Devrimsel, G., Metin, Y. \&Serdaroglu, Beyazal, M. Short-term effects of neuromuscular electrical stimulation and ultrasound therapies on muscle architecture and functional capacity in knee osteoarthritis: a randomized study. Clin Rehabil.33,418-427(2019).

15. Huang, M.H., Lin, Y.S., Lee, C.L. \&Yang, R.C. Use of ultrasound to increase effectiveness of isokinetic exercise for knee osteoarthritis. Arch Phys Med Rehabil. 86,1545-1551(2005).

16. Park, K., Hoffmeister, B., Han, D.K. \& Hasty, K. Therapeutic ultrasound effects on interleukin-1beta stimulated cartilage construct in vitro. Ultrasound Med Biol. 33,286-295(2007). 
17. Cakir, S. et al. Efficacy of therapeutic ultrasound for the management of knee osteoarthritis: a randomized, controlled, and double-blind study. Am J Phys Med Rehabil. 93,405-412 (2014).

18. Falconer, J., Hayes, K.W. \&Chang, R.W. Effect of ultrasound on mobility in osteoarthritis of the knee. A randomized clinical trial. Arthritis Care Res. 5,29-35(1992).

19. Ulus, Y.et al. Therapeutic ultrasound versus sham ultrasound for the management of patients with knee osteoarthritis: a randomized double-blind controlled clinical study. Int J Rheum Dis. 15,197-206(2012).

20. Draper, D.O., Knight, K., Fujiwara, T. \& Castel, J.C. Temperature change in human muscle during and after pulsed short-wave diathermy. J Orthop Sports Phys Ther. 29,13-18(1999).

21. Murray, C.C. \&Kitchen, S. Effect of pulse repetition rate on the perception of thermal sensation with pulsed shortwave diathermy. Physiother Res Int. 5,73-84 (2000).

22. Shields, N., Gormley, J. \& O'Hare, N. Short-wave diathermy: current clinical and safety practices. Physiother Res Int. 7,191-202 (2002).

23. Kitchen, S. \& Partridge, C. Review of shortwave diathermy continuous and pulsed patterns. Physiotherapy.78,243-252 (1992).

24. Jia, L., Chen, J., Wang, Y., Zhang, Y. \& Chen, W. Focused low-intensity pulsed ultrasound affects extracellular matrix degradation via decreasing chondrocyte apoptosis and inflammatory mediators in a surgically induced osteoarthritic rabbit model. Ultrasound Med Biol. 42,208-219(2016).

25. Huang, M.H., Yang, R.C., Lee, C.L., Chen, T.W. \&Wang, M.C. Preliminary results of integrated therapy for patients with knee osteoarthritis. Arthritis Rheum.53,812-820 (2005).

\section{Figures}

\section{Figure 1}

Procedure used for FLIPUS. The ST-35, EX-LE 4, medial and lateral knee joint spaces were marked, and the ultrasonic heads were fixed to ST 35, EX-LE 4, and the knee joint space.

\section{Figure 2}

Flowchart of eligible and recruited participants (CONSORT diagram).

FLIPUS: focused low-intensity pulsed ultrasound; PSWD: pulsed shortwave diathermy. 